\title{
Pricing Differentiated Services: A Game-Theoretic Approach
}

Eitan Altman ${ }^{\star 1}$, Dhiman Barman ${ }^{\star 2}$, Rachid El Azouzi*3, David Ros ${ }^{4}$, and Bruno Tuffin ${ }^{5}$

1 INRIA, BP 93, 06902 Sophia Antipolis Cedex, France

2111 Cummington Street, Dept. of C.S., Boston University, Boston, MA 02215, USA.

3 LIA/CERI, Univesité d'Avignon, Agroparc, BP 1228, 84911, Avignon, France

4 GET/ENST Bretagne, 35567 Cesson Sévigné Cedex, France

5 IRISA/INRIA, Campus Universitaire de Beaulieu, 35042 Rennes Cedex, France

\begin{abstract}
We consider TCP and real-time connections sharing a bottleneck buffer using RED. The latter gives differentiated services to the applications according to their choice of service class. The choice of a service class of an application depends both on the QoS as well as on the cost. We first study the performance of the system as a function of the connections' parameters and choice of service classes. We then study the decision problem of how to choose the service classes as a game. We finally study how to choose prices so that the resulting equilibrium would maximize the network's benefit.
\end{abstract}

Keywords: TCP, Buffer Management, RED/AQM, Nash equilibrium, Pricing, Economics

\section{Introduction}

We consider TCP connections as well as CBR (Constant Bit Rate) connections sharing a bottleneck link where a RED buffer management is used. We allow for service differentiation between the connections through the rejection probability which may depend on the connection. More specifically, we consider a buffer management scheme that uses a single averaged queue length to determine the rejection probabilities; for any given averaged queue size, packets belonging to connections with higher priority have smaller probability of being rejected. To obtain this differentiation we assume that the loss curve of RED is scaled by a factor that represents the priority level of the application. We obtain various performance measures of interest such as the throughput, the average queue size and the average drop probability.

We then address the question of the choice of priorities. Given utilities that depend on the performance measures and on the cost, the users are faced with

\footnotetext{
* The work of these authors was supported by France Telecom R\&D 001B001 contract.

** The work of this author was performed during internship at INRIA, financed by the INRIA's PrixNet ARC collaboration project
} 
a non-cooperative game. We establish conditions for an equilibrium to exist. We further provide conditions for convergence to equilibrium from non equilibria initial states. We finally study numerically the pricing problem of how the network should choose prices so that the resulting equilibrium would maximize its benefit.

We briefly mention some recent work in that area. Reference [5] has considered a related problem where the traffic generated by each session followed a Poisson process, and the service time was exponentially distributed. The decision variables were the input rates and the goodput was taken as the performance measure. The paper restricts to symmetric users and equilibria and pricing was not considered. In this framework, with a common RED buffer, it was shown that an equilibrium did not exist. An equilibrium was obtained and characterized for an alternative buffer management. We note that in contrast to [5], since we also include in the utility of CBR traffic a penalty for losses we do obtain an equilibrium when using RED. For other related papers, see $[1,3,7,9]$. Our pricing problem formulation is related to Stackelberg equilibrium $[4,6]$.

The structure of the paper is as follows: In Sec. 2 we describe the model and compute the performance for connections with given priority choices. Sec. 3 introduces the competition between connections at given prices, and concludes with the pricing problem. In Sec. 4 we study the game in the case of only CBR or only TCP connections. In Sec. 5 we provide an algorithm for computing the equilibrium for the symmetric case. Numerical examples are given in Sec. 6. We conclude the paper in Sec 7.

\section{The Model and Computation of Throughput}

RED is based on the following idea: there are two thresholds $q_{\min }$ and $q_{\max }$ such that the drop probability is 0 if the average queue length $q$ is less than $q_{\min }, 1$ if it is above $q_{\max }$, and $p(i)\left(x-q_{\min }\right) /\left(q_{\max }-q_{\min }\right)$ if it is $x$ with $q_{\min }<x<q_{\max }$; the latter is the congestion avoidance mode of operation. See Fig. 1.

Consider a set $\mathcal{N}$ containing $N$ TCP flows (or aggregate of flows) and a set $\mathcal{I}$ containing $I$ real-time flows that can be differentiated by RED; they all share a common buffer yet RED treats them differently. They all have common $q_{\min }$ and $q_{\max }$ but each flow $i$ may have a different $p(i)$, which is the value of the drop probability as the average queue tends to $q_{\max }$ (from the left). In other words, the slope $t_{i}$ of the linear part of the curve in Fig. 1 depends on the flow $i$ : $t_{i}=p(i) /\left(q_{\max }-q_{\min }\right)$. Denote $\mathbf{t}=\left(t_{i}, i \in \mathcal{I} \cup \mathcal{N}\right)$. We identify $t_{i}$ as the priority class of a connection. The service rate of the bottleneck router is given by $\mu$. We use the well-known relation for TCP rate:

$$
\lambda_{i}=\frac{1}{R_{i}} \sqrt{\frac{\alpha}{p_{i}}}, \quad i \in \mathcal{N},
$$

where $R_{i}$ and $p_{i}$ are TCP flow $i$ 's round trip time and drop probability, respectively. $\alpha$ is typically taken as $3 / 2$ (when the delayed ack option is disabled) or $3 / 4$ (when it is enabled). We shall assume throughout the paper that the queueing delay is negligible with respect to $R_{i}$ for the TCP connections. 


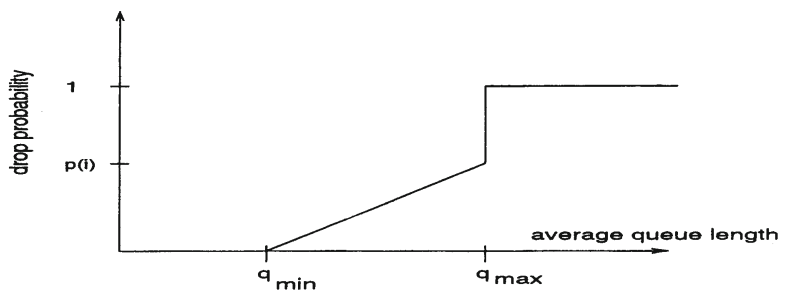

Fig. 1. Drop probability in RED as function $q$

In contrast, the rates $\lambda_{i}$, for $i \in \mathcal{I}$ are not controlled and are assumed to be fixed. If $\mathcal{N}=\emptyset$ we assume throughout the paper that $\sum_{j \in \mathcal{I}} \lambda_{j}>\mu$ (unless otherwise specified), otherwise the RED buffer is not a bottleneck. Similarly, if $\mathcal{I}=\emptyset$ we assume that TCP senders are not limited by the receiver window. In general, since the bottleneck queue is seen as a fluid queue, we can write $\sum_{j \in \mathcal{I} \cup \mathcal{N}} \lambda_{j}\left(1-p_{j}\right)=\mu$. If we operate in the linear part of the RED curve then this leads to the linear equations:

$$
\sum_{j \in \mathcal{I} \cup \mathcal{N}} \lambda_{j}\left(1-p_{j}\right)=\mu, \quad p_{i}=t_{i}\left(q-q_{\min }\right), \quad \forall i \in \mathcal{I} \cup \mathcal{N}
$$

with $(N+I+1)$ unknowns: $q$ (average queue length), and $p_{i}, i \in \mathcal{I} \cup \mathcal{N}$, where $\lambda_{i}, i \in \mathcal{N}$ is given by (1). Substituting (1) and

$$
p_{i}=t_{i}\left(q-q_{\min }\right) \forall i
$$

we obtain a single equation for $q$ :

$$
\sum_{j \in \mathcal{N}} \frac{1}{R_{j}} \sqrt{\frac{\alpha}{t_{j}\left(q-q_{\min }\right)}}\left(1-t_{j}\left(q-q_{\min }\right)\right)+\sum_{j \in \mathcal{I}} \lambda_{j}\left(1-t_{j}\left(q-q_{\min }\right)\right)=\mu .
$$

If we write $x=\sqrt{q-q_{\min }}$, then (3) can be written as a cubic equation in $x$ :

$$
\begin{gathered}
Z(x)=z_{3} x^{3}+z_{2} x^{2}+z_{1} x+z_{0}=0 \text { where } \\
z_{3}=\sum_{j \in \mathcal{I}} \lambda_{j} t_{j}, z_{2}=\sum_{j \in \mathcal{N}} \frac{1}{R_{j}} \sqrt{\alpha t_{j}}, z_{1}=\mu-\sum_{j \in \mathcal{I}} \lambda_{j}, z_{0}=-\sum_{j \in \mathcal{N}} \frac{1}{R_{j}} \sqrt{\frac{\alpha}{t_{j}}} .
\end{gathered}
$$

The equation has a unique positive solution if there are only TCP or only realtime connections; in either case, it becomes a quadratic equation.

Proposition 1 [2] Fix the values of $t_{j}, j \in \mathcal{I} \cup \mathcal{N}$. The cubic equation (4) has a unique real positive solution. Assume that the solution lies in the linear region of $R E D$. Then the average queue size is given as, $q=q_{\min }+x^{2}$ where $x$ is the unique positive solution of (4) and the loss probability for session $i$ is given by $p_{i}=t_{i}\left(q-q_{\min }\right)$. 
In the case of only CBR traffic operating in the linear region,

$$
\begin{aligned}
q & =q_{\min }+\frac{\sum_{j \in \mathcal{I}} \lambda_{j}-\mu}{\sum_{j \in \mathcal{I}} \lambda_{j} t_{j}} \text { and } \\
p_{i} & =t_{i} \frac{\sum_{j \in \mathcal{I}} \lambda_{j}-\mu}{\sum_{j \in \mathcal{I}} \lambda_{j} t_{j}}
\end{aligned}
$$

In the case of only TCP connections operating in the linear region, we have

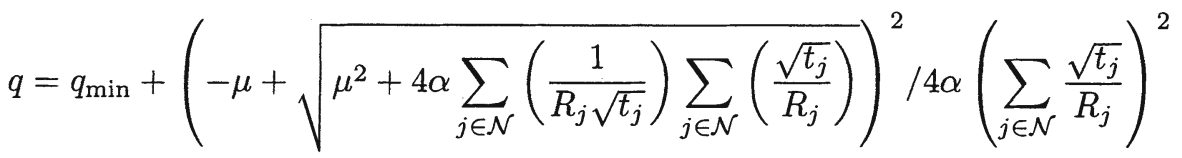

$$
\begin{aligned}
& p_{i}=t_{i}\left(-\mu+\sqrt{\mu^{2}+4 \alpha \sum_{j \in \mathcal{N}}\left(\frac{1}{R_{j} \sqrt{t_{j}}}\right) \sum_{j \in \mathcal{N}}\left(\frac{\sqrt{t_{j}}}{R_{j}}\right)}\right)^{2} / 4 \alpha\left(\sum_{j \in \mathcal{N}} \frac{\sqrt{t_{j}}}{R_{j}}\right)^{2} .
\end{aligned}
$$

\section{Utility, Pricing, and Equilibrium}

We denote a strategy vector by $\mathrm{t}$ for all flows such that $j$ th entry is $t_{j}$. By $\left(t_{i},[\mathbf{t}]_{-i}\right)$, we define a strategy where flow $i$ uses $t_{i}$ and all other flows $j \neq i$ use $t_{j}$ from vector $[\mathbf{t}]_{-i}$. We associate to flow $i$ a utility $U_{i}$. The utility will be a function of the QoS parameters and the price payed by flow $i$, and is determined by the actions of all flows. More precisely, $U_{i}\left(t_{i},[\mathbf{t}]_{-i}\right)$ is given by $a_{i} \lambda_{i}\left(1-p\left(t_{i},[\mathbf{t}]_{-i}\right)\right)$ $-b_{i} p\left(t_{i},[\mathbf{t}]_{-i}\right)-d\left(t_{i}\right)$ where the first term stands for the utility for the goodput, the second term stands for the dis-utility for the loss rate and the last term corresponds to the price $d\left(t_{i}\right)$ to be paid by flow $i$ to the network.

In particular, we find it natural to assume that a TCP flow $i$ has $b_{i}=0$ (as lost packets are retransmitted anyhow, and their impact is already taken into account in the throughput). Moreover, since $\lambda_{i}$ for TCP already includes the loss term $p_{i}\left(t_{i},[\mathbf{t}]_{-i}\right)$, the utility function of TCP is assumed to be $U_{i}\left(t_{i},[\mathbf{t}]_{-i}\right)=$ $a_{i} \lambda_{i}\left(1-p\left(t_{i},[\mathbf{t}]_{-i}\right)\right)-d\left(t_{i}\right)$.

We assume that the strategies or actions available to session $i$ are given by a compact set of the form: $t_{i} \in\left[t_{\text {min }}^{i}, t_{\text {max }}^{i}\right], i \in \mathcal{I} \cup \mathcal{N}$.

Each flow of the network strives to find its best strategy so as to maximize its own objective function. Nevertheless its objective function depends upon its own choice but also upon the choices of the other flows. In this situation, the solution concept widely accepted is the concept of Nash equilibrium.

Definition 1 A Nash equilibrium of the game is a strategy profile $\mathbf{t}=$ $\left(t_{1}, t_{2}, . ., t_{M}\right)$ where $M=I+N$ from which no flow have any incentive to deviate. More precisely, the strategy profile $\mathbf{t}$ is a Nash equilibrium, if the following holds true for any $i t_{i} \in \arg \max _{\bar{t}_{i} \in\left[t_{\text {min }}^{i}, t_{\text {max }}^{i}\right]} U_{i}\left(\bar{t}_{i},[\mathbf{t}]_{-\mathbf{i}}\right) . t_{i}$ is the best flow $i$ can do if the other flows choose the strategies $[\mathbf{t}]_{-i}$. 
Note that the network income is given by $\sum_{i \in \mathcal{I} \cup \mathcal{N}} d\left(t_{i}\right)$. Since the $p_{i}\left(t_{i},[\mathbf{t}]_{-i}\right)^{\prime}$ s are functions of $t_{i}$ and $[\mathbf{t}]_{-\mathbf{i}}, d$ can include pricing per volume of traffic successfully transmitted. In particular, we allow for $d$ to depend on the uncontrolled arrival rates of real-time sessions (but since these are constants, we do not make them appear as an argument of the function $d$ ).

We shall sometimes find it more convenient to represent the control action of connection $i$ as $T_{i}=1 / t_{i}$ instead of as $t_{i}$. Clearly, properties such as existence or uniqueness of equilibrium in terms of $t_{i}$ directly imply the corresponding properties with respect to $T_{i}$.

Optimal pricing: The goal here is to determine the pricing that maximizes the network's benefit; we seek to obtain a function $c\left(\mathbf{t}^{*}\right)=\arg \max _{d} \sum_{i=1}^{I} d\left(t_{i}^{*}\right)$, where $\mathbf{t}^{*}$ is a Nash equilibrium which can be obtained when considering special classes of function $d$. For instance, consider the set of functions $d(t)=d / e^{t}$. We then obtain a system of equations that can be solved numerically (to get the $t^{*}$ satisfying the Nash equilibrium). Then a numerical optimization over the parameter $d$ can be obtained. An assumption of this optimization problem is that the network knows the number of flows and the parameters $a_{i}, b_{i}$ and $R_{i} \forall i$. The optimal pricing will be done numerically in Section 6 .

\section{Equilibrium for Only CBR or Only TCP Connections}

We assume throughout that $t_{\max }^{i} \leq 1 /\left(q_{\max }-q_{\min }\right)$ for all connections. The bound for $t_{\text {max }}^{i}$ is given so that we have $t_{\max }^{i}\left(q_{\max }-q_{\min }\right) \leq 1$. From (2) we see that $p_{i} \leq 1$ with equality obtained only for the case $t_{i}=1 /\left(q_{\max }-q_{\min }\right){ }^{1}$

In our analysis, we are interested mainly in the linear region. For only realtime sessions or only TCP connections, we state the assumptions and describe the conditions for linear region operations and we show the existence of a Nash equilibrium. Proofs are given in [2].

Theorem 1 A sufficient condition for the system to operate in linear region is that for all $i$ : (a) For only real-time connections:

$$
\lambda>\mu \text { and } t_{\min }^{i}>\frac{\lambda-\mu}{\lambda\left(q_{\max }-q_{\min }\right)} .
$$

(b) Define $\lambda=\sum_{j \in \mathcal{I}} \lambda_{j}$ and $\Delta q:=q_{\max }-q_{\min }$. For only TCP connections:

$$
t_{\text {min }}^{i}>\left(\frac{-\mu+\sqrt{\mu^{2}+4 \alpha\left(\sum_{j \in \mathcal{N}} \frac{1}{R_{j}}\right)^{2}}}{4 \sqrt{\alpha \Delta q} \sum_{j \in \mathcal{N}} \frac{1}{R_{j}}}\right)^{2} .
$$

\footnotetext{
${ }^{1}$ Note that if the assumption does not hold then for some value $q^{\prime}<q_{\max }$ we would already have for some $i, p_{i}=1$ so one could redefine $q_{\max }$ to be $q^{\prime}$. An important feature in our model is that the queue length beyond which $p_{j}=1$ should be the same for all $j$.
} 
Theorem 2 [2, Appendix 10.2] Assume operation at the linear region of RED. Assume that the functions $d$ are convex in $T_{i}:=1 / t_{i}$. Then a Nash equilibrium exists.

Supermodular Games: In Thm 3 (resp. Thm 5) we present alternative conditions that provide sufficient conditions for a supermodular structure for real-time connections (resp. for only TCP connections). This implies in particular the existence of an equilibrium. Another implication of supermodularity is that a simple, so-called tatônnement or Round Robin scheme, for best responses converges to the equilibrium. To describe it, we introduce the following asynchronous dynamic greedy algorithm (GA).

Greedy Algorithm: Assume a given initial choice $\mathbf{t}^{\mathbf{0}}$ for all flows. At some strictly increasing times $\tau_{k}, k=1,2,3, \ldots$, flows update their actions; the actions $t_{i}^{k}$ at time $\tau_{k}>0$ are obtained as follows. A single flow $i$ at time $\tau_{k+1}$ updates its $t_{i}^{k+1}$ so as to optimize $U_{i}\left(.,\left[\mathbf{t}^{k}\right]_{-i}\right)$ where $\left[\mathbf{t}^{k}\right]_{-i}$ is the vector of actions of the other flows $j \neq i$. We assume that each flow updates its actions infinitely often. In particular, for the case of only real-time sessions, we update $t_{i}^{k+1}$ as follows:

$$
t_{i}^{k+1}=\underset{t_{i} \in\left[t_{\min }^{i}, t_{\text {max }}^{i}\right]}{\arg \max } a_{i} \lambda_{i}\left(1-p_{i}\right)-b_{i} p_{i}-d\left(t_{i}\right)
$$

where $p_{i}$ in (11) is given by (6). For TCP-only, we update $t_{i}^{k+1}$ as follows:

$$
t_{i}^{k+1}=\underset{t_{i} \in\left[t_{\min }^{i}, t_{\max }^{i}\right]}{\arg \max } \frac{a_{i}}{R_{i}} \sqrt{\frac{\alpha}{p_{i}}}\left(1-p_{i}\right)-d\left(t_{i}\right)
$$

where $p_{i}$ in (12) is given by (8).

Remark 1 For the case of real-time sessions, we could obtain a closed form solution for $t_{j}^{k+1}$ with specific cost function $d\left(t_{i}\right)$ such as $\frac{d}{t_{i}}$ which will lead to update of $t_{i}^{k+1}$ as follows,

$$
\delta_{i}^{k}=\frac{\sum_{j \neq i} \lambda_{j} t_{j}^{k}}{\sqrt{\left(a_{i} \lambda_{i}+b_{i}\right)\left(\sum_{j \in \mathcal{I}} \lambda_{j}-\mu\right)\left(\sum_{j \neq i} \lambda_{j} t_{j}^{k}\right)}-\lambda_{i} \sqrt{d}} \text { where } \delta_{i}^{k} \text { is such that }\left.\frac{\partial U_{i}}{\partial t_{i}}\right|_{t_{i}=\delta_{i}^{k}}=0 \text { and }
$$

$U_{i}$ corresponds to utility function of real-time session $i$. Then $t_{i}^{k+1}$ is given by :

$$
t_{i}^{k+1}= \begin{cases}t_{\min }^{i} & \text { if } \delta_{i}^{k}<0 \\ t_{\max }^{i} & \text { if } \delta_{i}^{k}<t_{\min }^{i}, \delta_{i}^{k} \geq 0 \\ t_{\min }^{i} & \text { if } \delta_{i}^{k}>t_{\max }^{i}, \delta_{i}^{k} \geq 0 \\ \delta_{i} & \text { otherwise. }\end{cases}
$$

The theorems below follow by establishing supermodularity of the games $[8,10]$, see [2] for detailed proofs.

Theorem 3 For the case of only real-time connections we assume that $\forall j$, $\lambda_{\min } \leq \lambda_{j} \leq \lambda_{\max }$, and $(I-1) \lambda_{\min } t_{\min } \geq \lambda_{\max } t_{\max }$, where $t_{\min }=\min _{i \in \mathcal{I}}\left\{t_{\min }^{i}\right\}$ 
and $t_{\max }=\max _{i \in \mathcal{I}}\left\{t_{\max }^{i}\right\}$. Then there is smallest equilibrium $\underline{t}$ and largest equilibrium $\overline{\mathbf{t}}$, and the $\mathbf{G A}$ dynamic algorithm converges to $\underline{\mathbf{t}}$ (resp. $\overline{\mathbf{t}}$ ) provided it starts with $t_{\min }^{j}$ for all $j$ (resp. $t_{\min }^{j}$ for all $j$ )

Theorem 4 For the case of only real-time connections, we assume that $\forall j, \lambda_{\min } \leq \lambda_{j} \leq \lambda_{\max }$, and $2 t_{\min }^{3} \lambda_{\min }^{2}>t_{\max }^{3} \lambda_{\max }^{2}$. Under supermodular condition, the Nash equilibrium exists and is unique.

Theorem 5 Case of only TCP connections: assume $\forall j, t_{\min } \leq t \leq t_{\max }$ and

$$
\left(3+p_{i}\right) \frac{\partial p_{i}}{\partial t_{i}} \frac{\partial p_{i}}{\partial t_{j}} \geq 2 p_{i}\left(p_{i}+1\right) \frac{\partial^{2} p_{i}}{\partial t_{i} \partial t_{j}} \forall i, j, i \neq j
$$

Then the game is super-modular and an equilibrium exists.

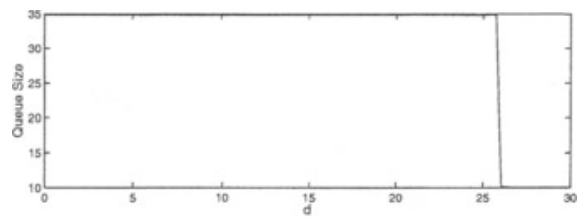

(a) queue size vs. $d$

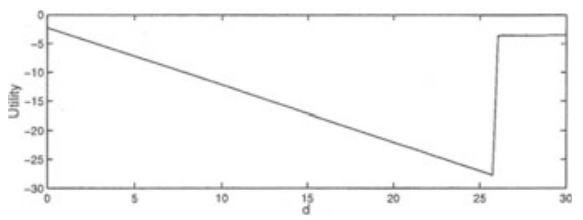

(c) utility vs. $d$

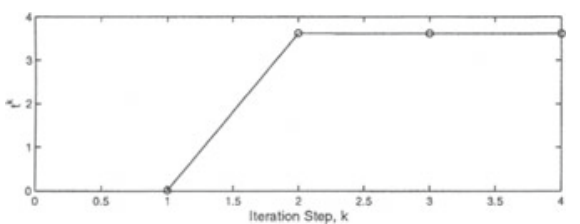

(e) $t^{0}=t_{\min }$

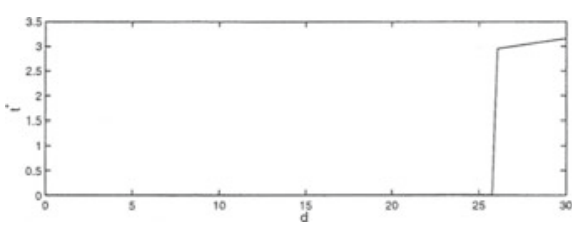

(b) $t^{*}$ vs. $d$

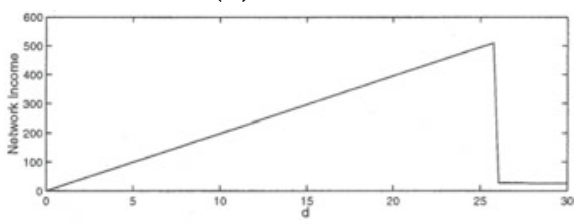

(d) network income vs. $d$

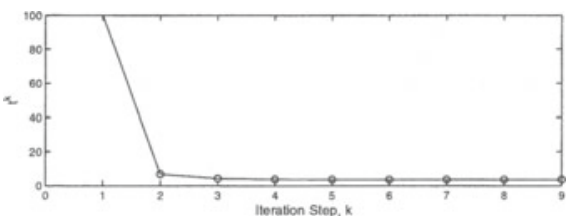

(f) $t^{0}=t_{\max }$

Fig. 2. Symmetric Real-Time flows: (a) queue size, (b) $t^{*}$ vs. $d$, (c) utility, (d) network income vs. $d$. (e), and (f): Convergence to Nash equilibrium

\section{Symmetric Users}

In this section, we assume that all flows have the same utility function (for all $i$, $a_{i}=a, \lambda_{i}=\bar{\lambda}$ and $b_{i}=b$ for real-time sessions and $a_{i}=a$ and $R_{i}=R$ for TCP connections) and the same intervals for strategies $\left(t_{\min }^{i}=t_{\min }\right.$ and $\left.t_{\max }^{i}=t_{\max }\right)$. 


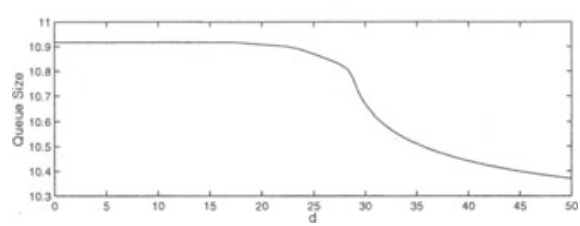

(a) queue Size vs. $d$

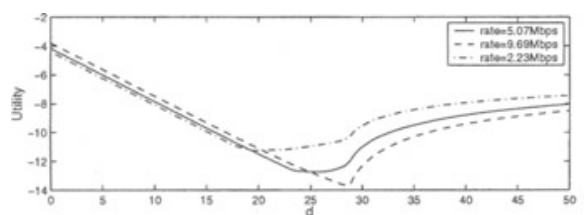

(c) utility vs. $d$

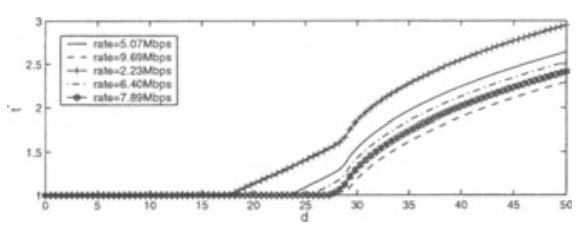

(b) $t^{*}$ vs. $d$

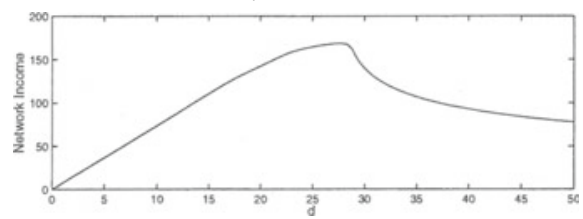

(d) network income vs. $d$

Fig. 3. Non-symmetric Real-Time flows: (a) queue size, (b) $t^{*}$ vs. $d$, (c) utility, and (d) network income vs. $d$

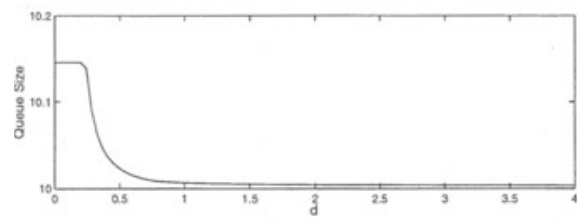

(a) queue Size vs. $d$

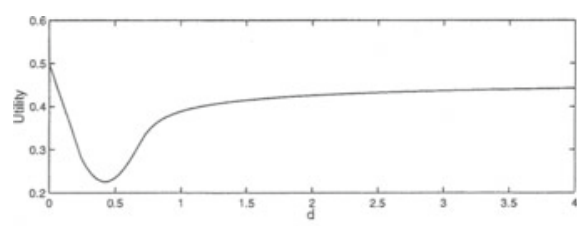

(c) TCP: utility vs $d$

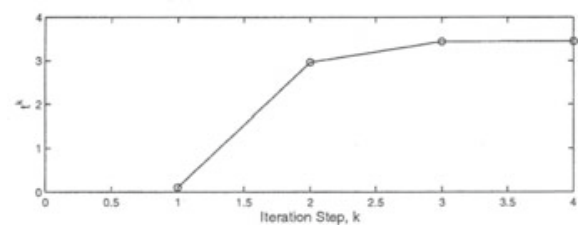

(e) $t^{0}=t_{\min }$

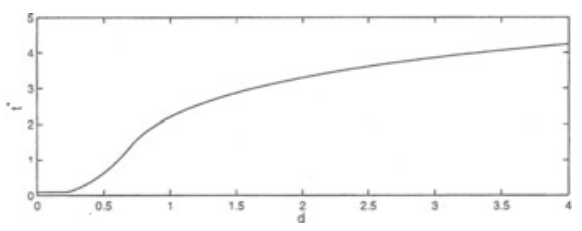

(b) $t^{*}$ vs. $d$

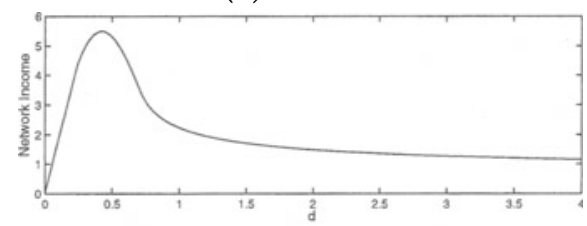

(d) TCP: network income vs. $d$

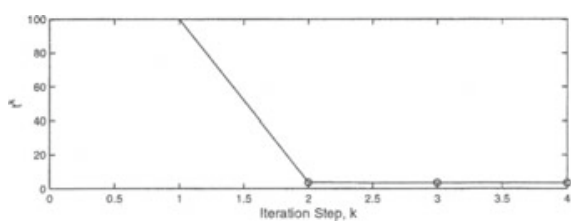

(f) $t^{0}=t_{\max }$

Fig. 4. Symmetric TCP flows: (a) queue size, (b) $t^{*}$ vs. $d$, (c) utility, (d) network income vs. $d, \mathrm{e}$ ) and $\mathrm{f}$ ) convergence to Nash equilibrium

\section{Algorithm for Symmetric Nash Equilibrium:}

For symmetric Nash equilibrium, we are interested in finding a symmetric equilibrium strategy $\mathbf{t}^{*}=\left(t^{*}, t^{*}, . ., t^{*}\right)$ such that for any flow $i$ and any strategy $t_{i}$ for that flow (real-time session or TCP connection),

$$
U\left(\mathbf{t}^{*}\right) \geq U\left(t_{i},\left[\mathbf{t}^{*}\right]_{-i}\right) .
$$




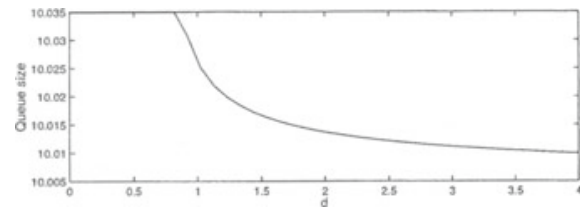

(a) queue Size vs. $d$

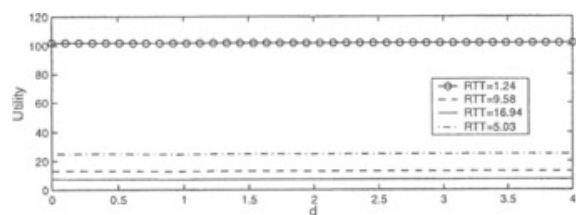

(c) utility vs $d$

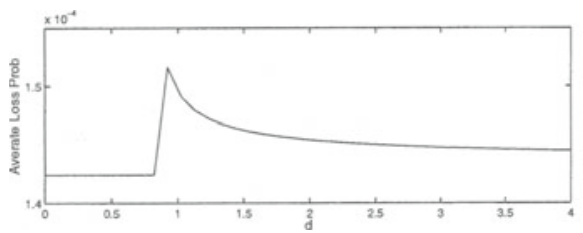

(e) Avg. Loss Prob. vs. $d$

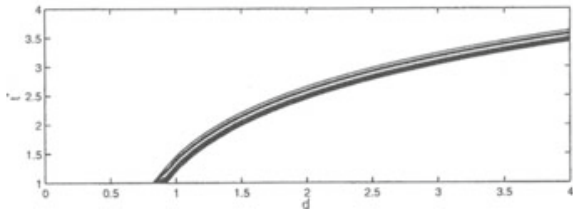

(b) $t^{*}$ vs. $d$

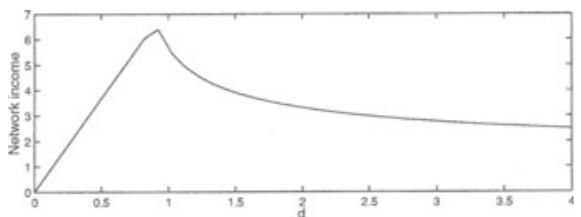

(d) network income vs. $d$

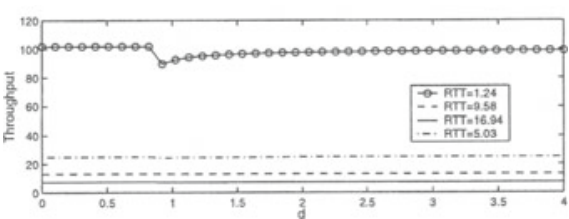

(f) Throughput vs. $d$

Fig. 5. Non-Symmetric TCP: (a) queue size, (b) $t^{*}$ vs $d$; (c) utility, (d) network pricing vs. $d$, (e) Avg. Loss prob, and (f) throughputs vs. $d$

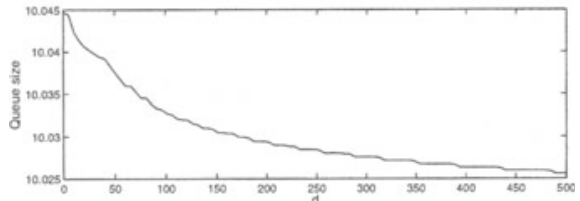

(a) queue Size vs. $d$

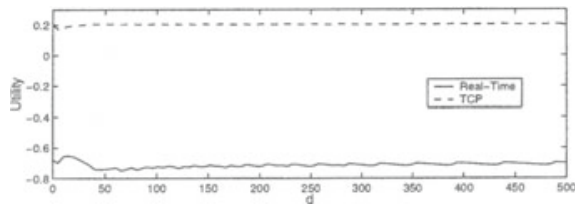

(c) utility vs. $d$

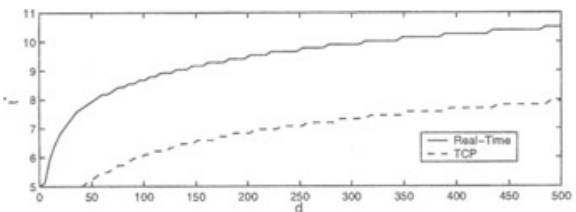

(b) $t^{*}$ vs. $d$

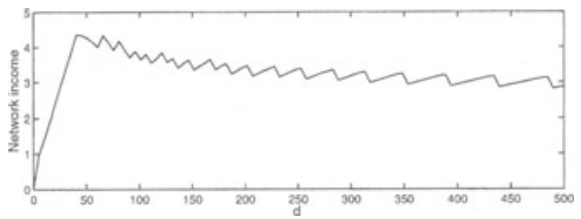

(d) network income vs. $d$

Fig. 6. Real-time and TCP: (a) utility and network income vs. $d$, b) $t^{*}$ vs. $d$, c) utility vs. $d$, and d) network income vs. $d$

Next we show how to obtain an equilibrium strategy. Due to symmetry, to see whether $\mathbf{t}^{*}$ is an equilibrium it suffices to check (14) for a single flow. We shall thus assume that there are $L+1$ flows all together, and that the first $L$ flows uses the strategy $\mathbf{t}^{o}=\left(t^{o}, \ldots, t^{o}\right)$ and flow $L+1$ use $t_{L+1}$. Define the set $\mathcal{Q}_{L+1}\left(\mathbf{t}^{\mathbf{o}}\right)=$ $\arg \max _{t_{L+1} \in\left[t_{\min }, t_{\max }\right]}\left(U\left(t_{L+1},\left[\mathbf{t}^{\mathbf{o}}\right]_{-(L+1)}\right)\right)$, where $\mathbf{t}^{\mathbf{o}}$ denotes (with some abuse 


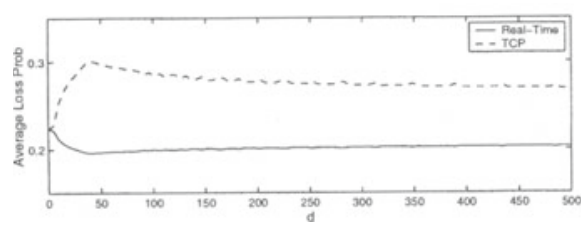

(a) Average loss prob vs $d$

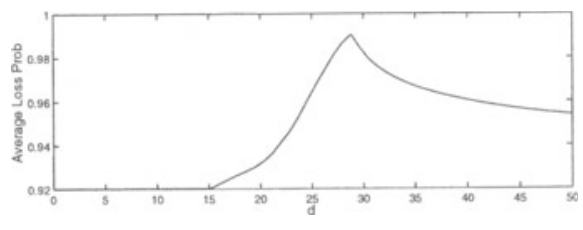

(b) Average loss prob vs $d$

Fig. 7. (a) Real-time and TCP, (b) Non-Symmetric Real-Time flows

of notation) the strategy where all flows use $t^{\circ}$, and where the maximization is taken with respect to $t_{L+1}$. Then $\mathbf{t}^{*}$ is a symmetric equilibrium if $t^{*} \in \mathcal{Q}_{L+1}\left(\mathbf{t}^{*}\right)$.

Theorem 6 [2] Consider real-time connections operating in linear region. The symmetric equilibrium $t^{*}$ satisfies: $\left.T^{*} \frac{\partial \hat{d}(T)}{\partial T}\right|_{T=T^{*}}=\frac{a \lambda+b}{(I \bar{\lambda})^{2}}$ where $T^{*}=1 / t^{*}$ and $\hat{d}(T)=d\left(\frac{1}{T}\right)$.

\section{$6 \quad$ Numerical Examples}

In the following simulations, we obtain a unique Nash equilibrium for only real-time sessions or only TCP connections without satisfying the conditions in Thm 4. Moreover, the GA algorithm converges without satisfying the conditions of supermodularity. All the conditions of supermodular games (Thm 3 and Thm 5) and uniqueness of Nash equilibrium (Thm 2 and Thm 3) are only sufficient but not necessary as shown in the numerical results.

The pricing function that we use for player $i$ throughout this section is $d / \exp \left(t_{i}\right)$. We shall investigate how the choice of the constant $d$ will affect the revenue of the network. ${ }^{2}$

Symmetric Real-Time flows: In the following numerical evaluations, we show the variation of different metrics as function of $d$. Fig. 2 corresponds to a unique symmetric equilibrium in which all the real-time flows have $\lambda_{i}=2 \mathrm{Mbps}$ with $t_{\min }=0.01, t_{\max }=100, \mathcal{I}=20, q_{\min }=10, q_{\max }=40, \mu=30 \mathrm{Mbps}$. Here we set the values of parameters to ensure that the system operates in linear region such as $t_{\min }>\frac{1}{\Delta q}\left(1-\frac{\mu}{\sum_{j \in \mathcal{I}} \lambda_{j}}\right)=0.0083$. The bound on $t_{\max }$ is needed only to limit the value of loss probability to 1 . The value of $d$ which maximizes the network revenue occurs at $d=25.75$. All the flows attain a loss rate of 0.25 . Note that for

\footnotetext{
${ }^{2}$ We note that it is desirable to have a "nontrivial" parameterized pricing function that leads to an optimal revenue for some parameter. We also tested other pricing functions that did turned out to be "trivial" in the sense that the benefit was always monotone in the parameter; an example of such a function is $\exp \left(-\beta t_{i}\right)$ and the network optimizes with respect to $\beta$.
} 
real-time flows symmetric case, $p_{i}^{*}=\left(\sum_{j \in \mathcal{I}} \lambda_{j}-\mu\right) / \sum_{j \in \mathcal{I}} \lambda_{j}$ at the Nash equilibrium is a constant. The average queue size, given by $q_{\min }+p_{i}^{*} / t_{i}^{*}$, is shown in Fig. 2 . We observe the value of $t^{*}$ at which maximum network income is achieved is close to $t_{\min }$ while the system operates in the linear region of RED throughout.

We plot in Fig. 2 (e,f) sample paths of a connection that uses the Algorithm for symmetric users (Sec. 5) (the evolution for all connections is the same). The figure shows convergence to the same Nash equilibrium when $t^{0}$ started from $t_{\min }$ or $t_{\max }$. We plot it for $d=41.525$. In Figure 2(e), the value of $t^{*}$ is 3.6163 , and in Figure 2(f), it is 3.6162 .

Non-symmetric real-time flows: In the next experiment (asymmetric) rates $\lambda_{i}$ are drawn uniformly from $[1,10] \mathrm{Mbps}$ with $t_{\min }=1, t_{\max }=100, q_{\max }=$ $40, q_{\min }=10, \mathcal{I}=20, \mu=30 \mathrm{Mbps}$. Figures 3 (a-d) show how different metrics vary with $d$ at unique Nash equilibrium. To ensure that the flows operate in linear region, we need $t_{\min }>\frac{1}{\Delta q} \geq \frac{1}{\Delta q}\left(1-\frac{\mu}{\sum_{j} \lambda_{j}}\right)$. We observe that $d=27.27$ maximizes the network revenue. Figure $3(\mathrm{~b})$ shows that values of $t^{*}$ for flows having higher rates increase slower than that of flows having lower rates, i.e., higher rate flows experience lower loss rates. Figure 3(c) shows that flows having different rates gain similarly in their utility functions. We plot the average loss rate in Figure 7(b). We confirm in these experiments about uniqueness of Nash equilibrium, although the sample path of different connections will depend on the connection rates.

Symmetric TCP Connections: For symmetric TCP connections we have considered $R_{i}=R=20 \mathrm{~ms}$ for all connections with $t_{\min }=0.1, t_{\max }=100, \mu=$ $30 \mathrm{Mbps}, N=20$. Figures $4(\mathrm{a}-\mathrm{f})$ show the corresponding figures. The maximum value of network revenue is found at $d=0.4040$. In this symmetric case, the loss probability is given by

$$
p^{*}=\frac{R^{2}}{3 N^{2}}\left\{\mu^{2}+\frac{3 N^{2}}{R^{2}}-\mu \sqrt{\mu^{2}+\frac{6 N^{2}}{R^{2}}}\right\}=0.0017
$$

To ensure that the symmetric TCP flows operate in the linear region, we satisfy the condition on $t_{\min }>\left(\frac{-\mu+\sqrt{\mu^{2}+4\left(\sum_{j \in \mathcal{N}} \frac{1}{R_{j}}\right)^{2}}}{4 \sqrt{\alpha \Delta q} \sum_{j \in N} \frac{1}{R_{j}}}\right)^{2}=4.6271 \times 10^{-5}$.

We plot sample paths of a connection which show convergence to Nash equilibrium when $t^{0}$ started from $t_{\min }$ or $t_{\max }$. We plot it for $d=3.821$. In Figure 4(e), the value of $t^{*}$ is 3.4480 , and in Figure $4(\mathrm{f})$, it is 3.4481 .

Non-symmetric TCP connections: We present a non-symmetric case in Figures $5(\mathrm{a}-\mathrm{f})$ in which $R_{i}$ s are drawn uniformly from $[1,20] \mathrm{ms}$ with $t_{\mathrm{min}}=$ $1, t_{\max }=100, \mu=30 \mathrm{Mbps}, N=20$. The value of $d$ at which network rev- 
enue is highest is 0.9321 . We ensure the operation in the linear region by setting $t_{\min }>\left(\frac{-\mu+\sqrt{\mu^{2}+4\left(\sum_{j \in \mathcal{N}} \frac{1}{R_{j}}\right)^{2}}}{4 \sqrt{\alpha \Delta q} \sum_{j \in \mathcal{N}} \frac{1}{R_{j}}}\right)^{2}=0.5476$.

Real-time connections and TCP flows: In this experiment, we combine both realtime and TCP connections. We have $I=15, N=15, \mu=13 \mathrm{Mbps}$, RTT $=10 \mathrm{~ms}$, $t_{\min }^{r e a l}=5, t_{\max }^{r e a l}=11, t_{\min }^{T C P}=5, t_{\max }^{T C P}=11, \lambda=1 \mathrm{Mbps}, q_{\min }=10, q_{\max }=40$. The highest network revenue is achieved at $d=40.40, t^{r e a l}=7.69, t^{T C P}=5$. In the simulations, we observe the values of $q<q_{\max }$ and since there is atleast one TCP flow $i$ with throughput, $\lambda_{i}>0$, it implies that the flow has loss probability, $p_{i}>0$ and average queue length, $q>q_{\min }$. We conclude that system operates in linear region. Our objective in this set of experiments is to show that there exists a Nash equilibrium for both real-time and TCP connections.

\section{Conclusions}

We have studied a fluid model of RED buffer management with different drop probabilities applied to both UDP and TCP traffic. We first computed the performance for fixed drop policies. We then investigated how the drop policies are determined using game theory and obtained and characterized the equilibrium. The equilibrium depends on the pricing strategy of the network provider. We finally addressed the problem of optimizing the revenue of the network provider.

\section{References}

1. T. Alpcan and T. Basar, "A game-theoretic framework for congestion control in a general topology networks", 41st IEEE CDC, Las Vegas, Nevada, Dec. 2002.

2. E. Altman, D. Barman, R. El Azouzi, D. Ros and B. Tuffin, "Pricing Differentiated Services: A Game-Theoretic Approach", INRIA Research report RR-4946, 2003.

3. E. Altman, T. Boulogne, R. El Azouzi, T. Jimenez and L. Wynter, "A survey on networking games", submitted. Available at http://www-sop.inria.fr/mistral/personnel/Eitan.Altman/ntkgame.html

4. T. Basar and R. Srikant, "A Stackelberg network game with a large number of followers", J. Optimization Theory and Applications, 115(3):479-490, 2002.

5. D. Dutta, A. Goel and J. Heidemann, "Oblivious AQM and Nash Equilibria", IEEE Infocom, 2003.

6. Y. A. Korilis, A. A. Lazar and A. Orda, "Achieving network optima using Stackelberg routing strategies", IEEE/ACM ToN, 5(1), pp. 161-173, 1997.

7. M. Mandjes, "Pricing strategies under heterogeneous service requirements", Computer Networks 42, pp. 231-249, 2003.

8. D. Topkis, "Equilibrium points in nonzero-sum n-person submodular games", SIAM J. Control and Optimization, 17:773-787, Nov. 1979.

9. Y. Jin and G. Kesidis, "Nash equilibria of a generic networking game with applications to circuit-switched networks", IEEE INFOCOM '03.

10. D. D. Yao, "S-modular games with queueing applications", Queueing Systems, 21:449-475, 1995. 